Artigo Original

\title{
Efeitos da reposição hidroeletrolítica sobre parâmetros cardiorrespiratórios em exercício e recuperação
}

\author{
Isadora Lessa Moreno ${ }^{1}$ \\ Carlos Marcelo Pastre ${ }^{1}$ \\ Marcelo Papoti $^{2}$ \\ Luiz Carlos Marques Vanderlei ${ }^{1}$ \\ ${ }^{1}$ Faculdade de Ciências e Tecnologia da UNESP - Universidade Estadual Paulista, \\ Departamento de Fisioterapia, Presidente Prudente, SP, Brasil \\ ${ }^{2}$ Faculdade de Ciências e Tecnologia da UNESP - Universidade Estadual Paulista, \\ Departamento de Educação Física, Presidente Prudente, SP, Brasil
}

\begin{abstract}
Resumo: Introdução: a necessidade de reposição ao máximo das perdas hídricas tornou-se estabelecida e difundida nos consensos internacionais. Entretanto, permanece pouco compreendida a influência da reposição quando administrada, igualmente, durante e após o exercício sobre parâmetros cardiorrespiratórios. Objetivo: analisar os efeitos da reposição hidroeletrolítica na frequência cardíaca (FC), pressão arterial sistólica (PAS), pressão arterial diastólica (PAD), saturação parcial de oxigênio $\left(\mathrm{SpO}_{2}\right)$ e frequência respiratória (f) de jovens durante e após um exercício de longa duração. Métodos: 31 jovens (21,55 $\pm 1,89$ anos) realizaram três visitas ao laboratório (intervalo de 48 horas entre elas), sendo na primeira aplicado um teste incremental, para determinação da carga utilizada nas visitas seguintes, e nas duas últimas, denominadas protocolo controle (PC) e protocolo experimental (PE), os sujeitos foram submetidos a 10 min de repouso supino, 90 min de exercício em esteira ergométrica ( $60 \%$ do $\mathrm{VO}_{2 p i c o}$ ) e 60 min de repouso supino. No PC não houve hidratação e no PE houve ingestão de solução isotônica. Os parâmetros $\mathrm{FC}, \mathrm{PAS}, \mathrm{PAD}, \mathrm{SpO}_{2}$ e f foram mensurados no final do repouso; nos minutos 30,60 e 90 do exercício, com exceção da f; e nos minutos $1,3,5,7,10,20,30,40,50$ e 60 pós-exercício. Foi aplicado o teste $t$ de Student ou teste de Mann-Whitney e ANOVA para medidas repetidas ou teste de Friedman seguidos de testes post hoc, com $p<0,05$. Resultados: a solução hidroeletrolítica proporcionou manutenção da PAS e da PAD, e menor incremento da FC durante o exercício; e promoveu retorno mais rápido da $\mathrm{FC}$ e conservou $\mathrm{PAD}, \mathrm{SpO}_{2}$, PAS (a partir do 5을 $\mathrm{min}$ e f (a partir do $30^{\circ} \mathrm{min}$ ) no período de recuperação. Conclusão: o protocolo de hidratação influenciou parâmetros cardiorrespiratórios de jovens durante e após a realização de atividade física submáxima de intensidade constante e longa duração.
\end{abstract}

Palavras-chave: Exercício aeróbico. Soluções de reidratação. Frequência cardíaca. Pressão arterial. Frequência respiratória.

\section{Effects of fluid replacement on cardiorespiratory parameters in exercise and recovery}

Abstract: Background: The replacement required of water waste is recognized and established in an international consensus. However, the influence of the replacement in cardiorespiratory parameters, especially when administered during and after physical activity, remains poorly understood. Objective: To analyze the effects of fluid replacement in heart rate (HR), systolic blood pressure (SBP), diastolic blood pressure (DBP), partial oxygen saturation ( $\mathrm{SpO} 2$ ) and respiratory rate $(\mathrm{f})$ of the youngsters during and after a long-term exercise. Methods: 31 individuals ( $21.55 \pm 1.89$ years old) performed three visits in the laboratory (48 hours among them), at first an incremental test was applied to determine the load used during subsequent visits. In the last two, called control protocol (CP) and the experimental protocol (EP), the subjects were undergone to a $10 \mathrm{~min}$ of supine rest, $90 \mathrm{~min}$ of treadmill exercise $\left(60 \%\right.$ of $\left.\mathrm{VO}_{2 \text { peak }}\right)$ and 60 min of supine rest. The difference between the protocols is that in the EP there was the intake isotonic solution and the CP there wasn't hydration. The parameters HR, SBP, DBP, $\mathrm{SpO}_{2}$ and $\mathrm{f}$ were measured at the end of the rest, in 30,60 and 90 minutes of the activity, except the f parameter, and at 1, 3, 5, 7, 10, 20, $30,40,50$ and 60 minute post-exercise. Student t test or Mann-Whitney, and ANOVA repeated measures or Friedman test followed by post hoc tests, were applied $(p<0.05)$. Results: the intake isotonic solution provided SBP and DBP maintenance and smaller increment in HR during the exercise; promoted faster return of $\mathrm{HR}$ and kept $\mathrm{SpO}_{2}$, SBP (from the 5th minute) and $f$ (from the 30th min) during recovery. Conclusion: the hydration protocol influenced the cardiorespiratory parameters for youngsters during and after submaximal physical exercise of constant intensity and long duration.

Keywords: Aerobic exercise. Rehydration solutions. Heart rate. Blood pressure. Respiratory rate. 
Considerando os desequilíbrios orgânicos promovidos pelo exercício e a importância do período de recuperação para reverter tais condições, faz-se importante o estudo dos processos envolvidos na restauração fisiológica quando solução hidroeletrolítica é utilizada como fonte de reposição durante e após um exercício de longa duração, visto que não foram encontrados estudos que avaliam o efeito dessa forma de administração sobre parâmetros cardiorrespiratórios. Hipotetiza-se que uma menor sobrecarga ao organismo e melhor recuperação dos parâmetros cardiorrespiratórios ocorram quando solução hidroeletrolítica é administrada durante e após um exercício de longa duração.

Dessa forma, o objetivo deste estudo é avaliar o comportamento da frequência cardíaca (FC), pressão arterial sistólica (PAS), pressão arterial diastólica (PAD), saturação parcial de oxigênio $\left(\mathrm{SpO}_{2}\right)$ e frequência respiratória (f) de jovens durante e após um exercício físico de longa duração com e sem a ingestão de solução hidroeletrolítica. responsável por reações bioquímicas intracelulares (GLAISTER, 2005; MARTINMÄKI e $\underline{\text { RUSKO}}$, 2008). A reorganização dessas funções é essencial para restauração de substratos utilizados durante o esforço físico e para que novos estímulos se procedam com reduzidos riscos à saúde e melhora da performance (COFFEY et al., 2004).

Além de proporcionar o bom funcionamento dos processos homeostáticos exigidos pelo exercício físico, a reposição de fluidos é uma importante estratégia para preservação do desempenho da atividade, uma vez que $2 \%$ de massa corporal perdida em decorrência da desidratação levam ao seu prejuízo (MONTAIN, 2008). Contudo, as características da solução de reposição devem respeitar fatores como duração e intensidade do evento desportivo, de modo que seja devidamente estimada a necessidade de reposição de água, eletrólitos e substrato energético (GISOLFI e DUCHMAN, 1992). Ainda, a reposição deve ocorrer antes, durante e após a sessão de exercício (GISOLFI e DUCHMAN, 1992), sendo já evidenciados efeitos benéficos, principalmente cardiovasculares, quando a hidratação é oferecida nesses períodos isoladamente (CARTER III et al., 2005; GONZÁLEZ-ALONSO et al., 1995; HAMILTON et al., 1991; VIANNA et al., 2008).

\section{Métodos}

\section{Sujeitos do estudo}

Trinta e um voluntários homens, jovens adultos e saudáveis, com média de idade de $21,55 \pm 1,89$ anos e ativos segundo o questionário internacional de atividade física (International physical activity questionnaire - IPAQ) (PARDINI et al., 2001). Não foram incluídos indivíduos tabagistas, que fizessem uso de medicamentos que influenciassem a atividade autonômica cardíaca, alcoólatras, portadores de distúrbios metabólicos e/ou endócrinos conhecidos e indivíduos sedentários, insuficientemente ativos e muito ativos segundo o IPAQ. Durante a execução do experimento não foram excluídos nenhum dos voluntários. O projeto foi submetido ao comitê de ética em pesquisa da Faculdade de Ciências e Tecnologia - FCT/UNESP, Campus de Presidente Prudente, processo o 168/07. Todos os voluntários foram informados dos objetivos e procedimentos do estudo e assinaram o termo de consentimento livre esclarecido conforme resolução 196/96 do Conselho Nacional de Saúde.

\section{Delineamento experimental}

Todos os voluntários realizaram três visitas ao laboratório, com intervalo mínimo de 48 horas entre elas, para realização dos seguintes 
procedimentos: Na primeira visita, foi aplicado um teste incremental, realizado em esteira ergométrica (Super ATL, Inbrasport, Brasil), de acordo com o protocolo máximo de Bruce (TEBEXRENI et al., 2001). Para realização do teste, inicialmente, os voluntários permaneceram em repouso na esteira em posição ortostática para estabilização dos valores iniciais dos parâmetros cardiorrespiratórios e, em seguida iniciaram o teste. Incentivo verbal foi utilizado na tentativa de obter um esforço físico máximo, sendo o teste interrompido mediante exaustão voluntária. Para determinação do consumo de oxigênio $\left(\mathrm{VO}_{2}\right)$, os gases expirados foram analisados por meio de um analisador metabólico calibrado regularmente (VO2000, Medical Graphics, St. Paul, MN, USA) (VIANNA et al., 2008). $O \mathrm{VO}_{2 \text { pico }}$ foi considerado o mais alto $\mathrm{VO}_{2}$ atingido no teste, sendo utilizado $60 \%$ desse valor para a intensidade da carga da esteira nos protocolos seguintes.

Nas visitas subsequentes, denominadas protocolos controle (PC) e experimental (PE), os voluntários foram submetidos a 10 minutos de repouso supino, seguidos por 90 minutos de exercício físico $\left(60 \%\right.$ do $\mathrm{VO}_{2 \text { pico }}$ ) e 60 minutos de recuperação. Além disso, no $\mathrm{PC}$ não houve administração de bebida isotônica, ao passo que no PE uma solução hidroeletrolítica (Gatorade, Brasil) foi administrada em 10 porções iguais e intervalos regulares de 15 minutos a partir do $15^{\circ}$ minuto de exercício até o final do período de recuperação.

Em todas as visitas, os voluntários foram orientados a não ingerir bebidas a base de cafeína por 24 horas antes, a consumir refeição leve à base de frutas duas horas antes do teste, a evitar esforços físicos vigorosos no dia anterior e a estarem trajados com roupas adequadas e confortáveis ao esforço físico (bermuda, camiseta, tênis e meias).

\section{Protocolos controle e experimental}

Os protocolos foram realizados em uma sala com temperatura média de $26,0 \pm 2,34 \stackrel{\circ}{\circ} \mathrm{e}$ umidade de $55,13 \pm 10,40 \%$, entre 15 e 18 horas para evitar a variação circadiana. A fim de garantir a condição de hidratação inicial, os voluntários ingeriram $500 \mathrm{ml}$ de água, duas horas antes de iniciar ambos os protocolos (HERNANDEZ e NAHAS, 2009).
Ao chegarem ao local da coleta foram verificados: peso, estando os voluntários desnudos (balança digital Plenna, TIN 00139 MAXIMA, Brasil) e altura (estadiômetro ES 2020 Sanny, Brasil). Em seguida, foi posicionada sobre a região do precórdio a cinta de captação e no punho o receptor de FC (Polar Electro - S810i, Kempele, Finland) para registro desse parâmetro. Os voluntários permaneceram em repouso supino por 10 minutos e imediatamente após a temperatura axilar (termômetro BD Thermofácil, China) foi mensurada. Ao final desse tempo foram mensuradas FC, PAS, PAD, f e $\mathrm{SpO}_{2}$.

Para aferição da pressão arterial utilizou-se um estetoscópio (Littmann, Saint Paul, USA) e esfigmomanômetro aneróide (Welch Allyn Tycos, New York, USA) no braço esquerdo dos voluntários, de acordo com os critérios estabelecidos pela $\mathrm{VI}$ Diretrizes Brasileiras de Hipertensão (2010). As medidas de $f$ foram realizadas pela contagem das respirações durante um minuto sem que o voluntário tomasse conhecimento do processo, para que as características usuais da respiração não fossem modificadas; e a $\mathrm{SpO}_{2}$ foi verificada por meio de um oxímetro de pulso (Mindray PM-50 Pulse Oximeter, China).

Em seguida, os voluntários executaram 90 minutos de exercício em esteira ergométrica $(60 \%$ do $\mathrm{VO}_{2 \text { pico }}$ ) e nos minutos 30,60 e 90 foram mensuradas $\mathrm{FC}, \mathrm{PAS}, \mathrm{PAD}$ e $\mathrm{SpO}_{2}$. Ao término do exercício, eles foram colocados em repouso supino, para dar início aos 60 minutos de recuperação, e a temperatura axilar foi novamente verificada. Os parâmetros FC, PAS, $\mathrm{PAD}, \mathrm{SpO}_{2}$ e f foram mensurados nos minutos 1 , $3,5,7,10,20,30,40,50$ e 60 da recuperação. Ao final desse tempo, os voluntários mensuraram seu peso estando novamente desnudos.

A quantidade de solução hidroeletrolítica administrada durante o exercício físico e a recuperação no $\mathrm{PE}$ foi baseada na diferença do peso corporal mensurado antes e ao final do PC, portanto o mesmo foi aplicado antes do PE. Essa técnica implica que um grama de massa corporal perdido equivale a um mililitro de líquido perdido (VON DUVILLARD et al., 2004). Ainda, no PE, a mensuração dos parâmetros cardiorrespiratórios nos momentos coincidentes ocorreu imediatamente após a ingestão da solução. Para confirmar a condição de hidratação ao final do $\mathrm{PE}$, os voluntários colheram e analisaram a urina 
(Choiceline 10, Roche ${ }^{\circledR}$, Brasil) após a mensuração do peso corporal final. A partir da densidade específica da urina, utilizada como marcador de nível de hidratação, foram considerados euidratados indivíduos com densidade entre 1,013 e 1,029 e desidratados com valores maior ou igual a 1,030 (ARMSTRONG, 2005).

\section{Procedimentos estatísticos}

A normalidade dos dados foi determinada usando o teste Shapiro-Wilk. Para comparação das variáveis entre PC e PE no mesmo tempo de análise, foi aplicado o teste $t$ de Student para dados não pareados ou o teste de Mann-Whitney. Para comparação entre os momentos do mesmo protocolo foi aplicada a análise de variância para medidas repetidas ou teste de Friedman seguidos, respectivamente, dos testes post hoc de Bonferroni e Dunn. A significância estatística foi fixada em $5 \%$ para todas as análises e os cálculos foram realizados utilizando o software comercial Minitab - versão 13.20 (Minitab, PA., USA). O cálculo do poder do estudo, com o número de sujeitos analisados e nível de significância de $5 \%$ (teste bi-caudal), garantiu um poder do teste superior a $80 \%$ para detectar diferenças entre as variáveis.

\section{Resultados}

As características antropométricas dos sujeitos bem como as respostas obtidas no teste incremental estão descritas na tabela 1 .

Os valores obtidos das mensurações do peso e da temperatura corporal no PC e PE estão descritos na tabela 2.

Tabela 1- Valores médios, seguidos pelos seus respectivos desvios-padrão, valores mínimo e máximo dos dados antropométricos e do teste incremental dos sujeitos analisados.

\begin{tabular}{|c|c|c|}
\hline Variáveis & Média \pm DP & Mínimo/Máximo \\
\hline \multicolumn{3}{|l|}{ Dados antropométricos } \\
\hline Idade (anos) & $21,55 \pm 1,89$ & [18 - 25] \\
\hline Peso $(\mathrm{kg})$ & $72,62 \pm 11,54$ & {$[53,8-95,3]$} \\
\hline Altura (m) & $1,77 \pm 0,08$ & {$[1,60-1,94]$} \\
\hline IMC $\left(\mathrm{kg} / \mathrm{m}^{2}\right)$ & $23,00 \pm 2,83$ & {$[16,87-28,07]$} \\
\hline \multicolumn{3}{|l|}{ Teste de esforço máximo } \\
\hline $\mathrm{VO}_{2 \text { pico }}\left(\right.$ I. $\left.\mathrm{min}^{-1}\right)$ & $3,37 \pm 0,60$ & {$[2,02-5,14]$} \\
\hline $60 \% \mathrm{VO}_{2 \text { pico }}\left(\mathrm{I} \cdot \mathrm{min}^{-1}\right)$ & $2,02 \pm 0,36$ & {$[1,21-3,08]$} \\
\hline $\mathrm{FC}(\mathrm{bpm})$ & $160,74 \pm 10,76$ & {$[139-179]$} \\
\hline
\end{tabular}

Tabela 2- Valores médios, seguidos pelos seus respectivos desvios-padrão, valores mínimo e máximo das medidas de massa corporal e temperatura corporal nas condições controle e experimental.

\begin{tabular}{cccc}
\hline Variável & Tempo & Protocolo controle & Protocolo experimental \\
\hline & Inicial & $73,03 \pm 11,56$ & $72,90 \pm 11,50$ \\
Peso corporal $(\mathbf{k g})$ & & {$[54,70-96,10]$} & {$[53,50-96,60]$} \\
& Final & $71,55 \pm 11,30$ & $73,08 \pm 11,51$ \\
& & {$[53,60-94,20]$} & {$[53,50-97,00]$} \\
\hline Temperatura corporal $\left({ }^{\circ} \mathbf{C}\right)$ & Antes & $36,44 \pm 0,47$ & $36,30 \pm 0,37$ \\
& & {$[35,00-38,00]$} & {$[35,00-36,90]$} \\
& \multirow{2}{*}{ Após exercício } & $37,20 \pm 0,53$ & $36,83 \pm 0,42$ \\
& & {$[35,50-38,00]$} & {$[36,00-38,00]$} \\
\hline
\end{tabular}

Legenda: $\mathrm{kg}=$ quilograma; ${ }^{\circ} \mathrm{C}=$ graus Celsius.

Foi observada perda de peso e aumento de temperatura corporal quando os indivíduos encontravam-se hipoidratados (tabela 2). A porcentagem da massa corporal perdida pelos voluntários ao final do $P C$ foi de $2,02 \pm 0,61 \%$, sendo o consumo médio de solução administrada no PE igual a $1,48 \pm 0,54 \mathrm{I}$. A densidade específica da urina $(1,018 \pm 0,004)$, avaliada ao final do $\mathrm{PE}$, confirma que o volume de solução administrado foi suficiente para manter o estado euidratado dos sujeitos.

A figura 1 mostra o comportamento da FC durante o período de exercício (Figura 1A) e recuperação (Figura 1B) e sua comparação em relação ao repouso inicial no PC e PE. Durante o exercício, a FC aumentou progressivamente em 
ambos os protocolos, contudo, embora não significante, o incremento foi menor no PE em comparação ao PC. No período de recuperação, a FC reduziu mais lentamente no $P C$ em comparação ao $\mathrm{PE}$, sendo essa diferença estatisticamente significante a partir do terceiro minuto $(99,77 \pm 11,50$ vs. 93,09 $\pm 9,40$ bpm; $p<$ $0,05)$ e mantida até o final do período de recuperação $(84,12 \pm 11,69$ vs. 78,67 \pm 9,27 bpm; $\mathrm{p}<0,05)$. Em ambos os protocolos, ao final de 60 minutos de recuperação, a FC não retornou ao valor basal.
A

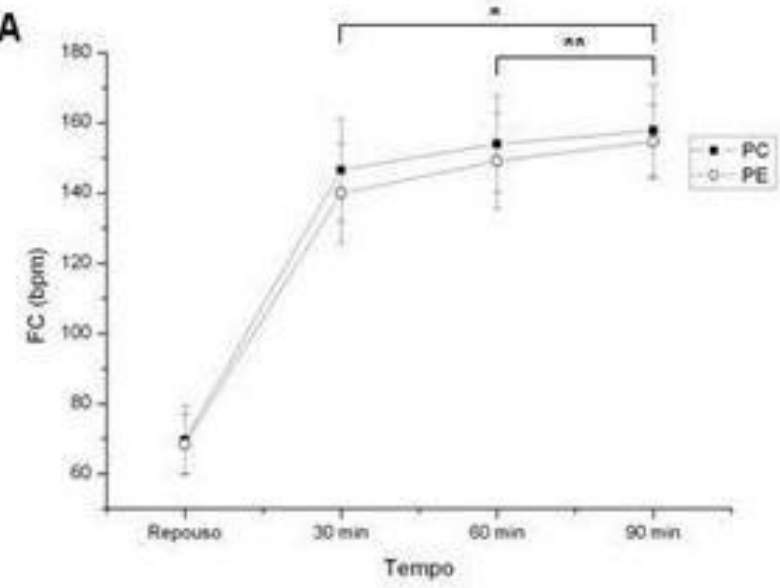

B

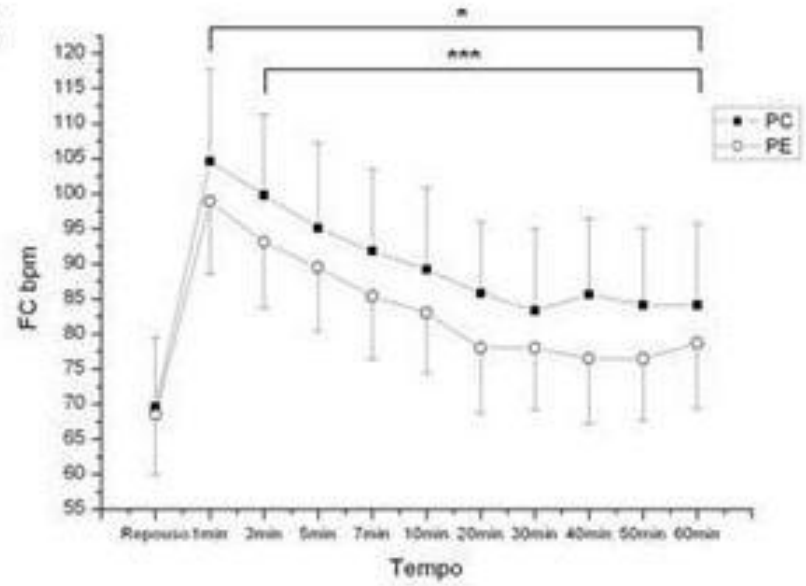

Legenda: bpm = batimentos por minutos; $\min =$ minuto

* Valor com diferença estatística em relação ao repouso (ANOVA para medidas repetidas seguida do teste de Bonferroni; $p<0,05)$;

** Valor com diferença estatística em relação ao tempo 30 min (ANOVA para medidas repetidas seguida do teste de Bonferroni; $p<0,05)$;

*** Valor com diferença estatística na análise interprotocolos (Teste $t$ de Student para dados não pareados; $p<0,05$ ).

Figura1- Comportamento da frequência cardíaca $(F C)$ durante o período de exercício (Figura $1 A)$ e recuperação (Figura 1B) e sua comparação em relação ao repouso inicial nos protocolos controle (PC) e experimental (PE). Valores expressos como média desvios padrões.

Tabela 3- Valores médios, seguidos dos seus respectivos desvios padrões, mediana e intervalo de confiança a $95 \%$ de PAS, PAD e $\mathrm{SpO}_{2}$ obtidos nos protocolos controle e experimental em repouso e durante o exercício.

\begin{tabular}{|c|c|c|c|c|c|}
\hline \multirow{2}{*}{ Parâmetro } & \multirow{2}{*}{ Protocolo } & \multicolumn{4}{|c|}{ Tempo } \\
\hline & & Repouso & $30 \mathrm{~min}$ & $60 \mathrm{~min}$ & $90 \mathrm{~min}$ \\
\hline \multirow{2}{*}{ PAS (mmHg) } & Controle & $\begin{array}{c}116,12 \pm 10,14^{a} \\
(120,0) \\
{[112,41-119,85]}\end{array}$ & $\begin{array}{c}144,19 \pm 14,55^{b} \\
(140,0) \\
{[138,86-149,53]}\end{array}$ & $\begin{array}{c}138,70 \pm 11,47 \\
(140,0) \\
{[134,50-142,92]}\end{array}$ & $\begin{array}{c}137,09 \pm 10,70 \\
(140,0) \\
{[133,17-141,02]}\end{array}$ \\
\hline & Experimental & $\begin{array}{c}113,54 \pm 8,38^{a} \\
(110,0) \\
{[110,47-116,62]}\end{array}$ & $\begin{array}{c}140,96 \pm 11,93 \\
(140,0) \\
{[136,59-145,34]}\end{array}$ & $\begin{array}{c}140,64 \pm 11,52 \\
(140,0) \\
{[136,42-144,87]}\end{array}$ & $\begin{array}{c}140,32 \pm 13,03 \\
(140,0) \\
{[135,54-145,10]}\end{array}$ \\
\hline \multirow{2}{*}{ PAD (mmHg) } & Controle & $\begin{array}{c}75,87 \pm 9,47^{a} \\
(80,0) \\
{[72,39-79,34]}\end{array}$ & $\begin{array}{c}69,03 \pm 9,07^{c} \\
(70,0) \\
{[65,70-72,36]}\end{array}$ & $\begin{array}{c}65,48 \pm 7,67 \\
(60,0) \\
{[62,66-68,29]}\end{array}$ & $\begin{array}{c}63,87 \pm 8,43 \\
(60,0) \\
{[60,77-66,96]}\end{array}$ \\
\hline & Experimental & $\begin{array}{c}75,12 \pm 8,26^{a} \\
(70,0) \\
{[72,09-78,16]}\end{array}$ & $\begin{array}{c}67,41 \pm 8,55 \\
(70,0) \\
{[64,28-70,55]}\end{array}$ & $\begin{array}{c}67,74 \pm 10,23 \\
(70,0) \\
{[63,98-71,49]}\end{array}$ & $\begin{array}{c}67,09 \pm 7,82 \\
(70,0) \\
{[64,22-69,96]}\end{array}$ \\
\hline \multirow[b]{2}{*}{$\mathrm{SpO}_{2}(\%)$} & Controle & $\begin{array}{c}97,12 \pm 0,88^{c} \\
(97,0) \\
{[96,80-97,45]}\end{array}$ & $\begin{array}{c}96,87 \pm 0,84 \\
(97,0) \\
{[96,56-97,18]}\end{array}$ & $\begin{array}{c}96,74 \pm 0,77 \\
(97,0) \\
{[96,45-97,02]}\end{array}$ & $\begin{array}{c}96,54 \pm 0,85 \\
(97,0) \\
{[96,23-96,86]}\end{array}$ \\
\hline & Experimental & $\begin{array}{c}97,45 \pm 0,67^{c} \\
(97,0) \\
{[97,20-97,69]}\end{array}$ & $\begin{array}{c}97,16 \pm 0,68 \\
(97,0) \\
{[96,90-97,41]}\end{array}$ & $\begin{array}{c}97,06 \pm 0,77 \\
(97,0) \\
{[96,78-97,34]}\end{array}$ & $\begin{array}{c}96,83 \pm 0,89 \\
(97,0) \\
{[96,50-97,16]}\end{array}$ \\
\hline \multicolumn{6}{|c|}{$\begin{array}{l}\text { Legenda: } \mathrm{PAS}=\text { pressão arterial sistólica; } \mathrm{PAD}=\text { pressão arterial diastólica; } \mathrm{FC}=\text { frequência cardíaca; } \mathrm{SpO} \mathrm{O}_{2}= \\
\text { saturação parcial de oxigênio; } \mathrm{mmHg}=\text { milímetros de mercúrio; } \mathrm{bpm}=\text { batimentos por minuto; } \text { min = minuto. }\end{array}$} \\
\hline \multicolumn{6}{|c|}{$\begin{array}{l}{ }^{a} \text { Valor com diferença estatística em relação aos tempos } 30,60 \text { e } 90 \text { min (ANOVA para medidas repetidas seguida } \\
\text { do teste de Bonferroni; } p<0,05 \text { ); }{ }^{b} \text { Valor com diferença estatística em relação aos tempos } 60 \text { e } 90 \text { min (ANOVA } \\
\text { para medidas repetidas seguida do teste de Bonferroni; } p<0,05) ;{ }^{c} \text { Valor com diferença estatística em relação ao } \\
\text { tempo } 90 \text { min (ANOVA para medidas repetidas seguida do teste de Bonferroni; } p<0,05 \text { ). }\end{array}$} \\
\hline
\end{tabular}


A tabela 3 mostra os valores de PAS, PAD e $\mathrm{SpO} 2$ obtidos nos protocolos em repouso e durante o exercício. A PAS se elevou em exercício comparado ao repouso, em ambos os protocolos, e entre 30 e 90 minutos exibiu queda de $5 \%$ no $P C(144,19 \pm 14,55$ vs. $137,09 \pm 10,70$ mmHg; $p<0,05)$, não observada no PE. A PAD reduziu durante 0 exercício comparado ao repouso, em ambos os protocolos, e entre 30 e 90 minutos exibiu queda de $7,5 \%$ no PC $(69,03 \pm$ $9,07$ vs. $63,87 \pm 8,43 \mathrm{mmHg} ; p<0,05)$ não observada no PE. Quanto à $\mathrm{SpO}_{2}$, diferenças significantes foram observadas entre o repouso e o 90 minutos, em ambos os protocolos.

A tabela 4 mostra os valores de PAS e PAD obtidos em repouso e recuperação no PC e PE. Valores significantemente maiores de PAS foram observados quando comparado o primeiro minuto ao repouso, tanto no $\mathrm{PC}(127,03 \pm 12,63$ vs. $116,12 \pm 10,14 \mathrm{mmHg} ; \mathrm{p}<0,05)$ quanto no $\mathrm{PE}$ $(125,22 \pm 11,57$ vs. $113,54 \pm 8,38 \mathrm{mmHg} ; \mathrm{p}<$
$0,05)$ e o terceiro minuto de recuperação ao repouso $(119,61 \pm 11,11$ vs. $113,54 \pm 8,38 \mathrm{mmHg}$; $\mathrm{p}<0,05)$ no PE. No PC, a partir do minuto 30 de recuperação, a PAS exibiu valores significantemente menores quando comparados ao repouso, não observado no PE. Para PAD, diferenças significantes foram observadas apenas no $\mathrm{PC}$ quando comparado o repouso aos minutos 1, 3, 5, 30 e 40 da recuperação.

$\mathrm{Na}$ tabela 5 observa-se os valores de SpO2 ef em repouso e recuperação no $\mathrm{PC}$ e $\mathrm{PE}$. Para a $\mathrm{SpO}_{2}$, em todos os momentos da recuperação, os valores foram menores no $\mathrm{PC}$ quando comparado ao PE $(p<0,05)$. Diferenças significantes foram também observadas no PC quando comparado o repouso aos minutos 7, 10, 20 e 30. Quanto à f, avaliada apenas no período de recuperação, valores significantemente maiores foram observados quando comparados os minutos 1,3 , 5,7 e 10 ao repouso no $\mathrm{PC}$, e nos minutos $1,3,5$, 7,10 e 20 ao repouso no $P E$.

Tabela 4- Valores médios, seguidos dos seus respectivos desvios-padrão, mediana e intervalo de confiança a 95\% de PAS e PAD obtidos nos protocolos controle e experimental em repouso e durante o período de recuperação.

\begin{tabular}{|c|c|c|c|c|}
\hline \multirow{2}{*}{ Tempo } & \multicolumn{2}{|c|}{ PAS (mmHg) } & \multicolumn{2}{|c|}{ PAD (mmHg) } \\
\hline & Controle & Experimental & Controle & Experimental \\
\hline \multirow{2}{*}{ Repouso } & $116,12 \pm 10,14(120,0)$ & $113,54 \pm 8,38(110,0)$ & $75,87 \pm 9,47(80,0)$ & $75,12 \pm 8,26(70,0)$ \\
\hline & {$[112,41-119,85]$} & {$[110,47-116,62]$} & {$[72,39-79,34]$} & {$[72,09-78,16]$} \\
\hline \multirow{2}{*}{$1 \mathrm{~min}$ rec } & $127,03 \pm 12,63(120,0)^{a}$ & $125,22 \pm 11,57(128,0)^{a}$ & $69,90 \pm 9,10(70,0)^{a}$ & $72,96 \pm 8,94(70,0)$ \\
\hline & {$[122,40-131,67]$} & {$[120,98-129,47]$} & {$[66,56-73,24]$} & {$[69,68-76,24]$} \\
\hline \multirow{2}{*}{$3 \mathrm{~min}$ rec } & $119,41 \pm 11,76(120,0)$ & $119,61 \pm 11,11(120,0)^{a}$ & $71,22 \pm 11,25(70,0)^{a}$ & $74,58 \pm 9,00(74,0)$ \\
\hline & {$[115,10-123,73]$} & {$[115,54-123,69]$} & {$[67,10-75,35]$} & {$[71,27-77,88]$} \\
\hline \multirow{2}{*}{$5 \mathrm{~min} \mathrm{rec}$} & $117,03 \pm 10,89(120,0)$ & $116,12 \pm 11,45(120,0)$ & $70,96 \pm 10,81(70,0)^{a}$ & $74,45 \pm 9,91(78,0)$ \\
\hline & {$[113,04-121,03]$} & {$[111,93-120,33]$} & {$[67,00-74,93]$} & {$[70,81-78,08]$} \\
\hline \multirow{2}{*}{$7 \mathrm{~min}$ rec } & $116,38 \pm 11,28(110,0)$ & $115,61 \pm 11,27(120,0)$ & $73,22 \pm 9,40(70,0)$ & $74,96 \pm 8,76(78,0)$ \\
\hline & {$[110,51-119,16]$} & {$[111,48-119,75]$} & {$[69,77-76,67]$} & {$[71,75-78,18]$} \\
\hline \multirow{2}{*}{$10 \mathrm{~min}$ rec } & $114,83 \pm 11,79(110,0)$ & $114,58 \pm 10,96(118,0)$ & $74,06 \pm 8,30(76,0)$ & $75,09 \pm 9,49(76,0)$ \\
\hline & {$[110,51-119,16]$} & {$[110,56-118,60]$} & {$[71,02-77,10]$} & {$[71,61-78,57]$} \\
\hline \multirow{2}{*}{$20 \mathrm{~min}$ rec } & $113,48 \pm 10,95(110,0)$ & $112,25 \pm 11,16(110,0)$ & $73,48 \pm 9,29(70,0)$ & $74,58 \pm 8,00(70,0)$ \\
\hline & {$[109,47-117,50]$} & {$[108,16-116,35]$} & {$[70,07-76,89]$} & {$[71,64-77,51]$} \\
\hline \multirow{2}{*}{$30 \mathrm{~min}$ rec } & $110,96 \pm 12,47(110,0)^{a}$ & $112,45 \pm 10,33(110,0)$ & $71,67 \pm 9,42(70,0)^{a}$ & $75,48 \pm 9,15(70,0)$ \\
\hline & {$[106,39-115,54]$} & {$[108,66-116,24]$} & {$[68,22-75,13]$} & {$[72,12-78,84]$} \\
\hline \multirow{2}{*}{$40 \mathrm{~min}$ rec } & $110,83 \pm 13,04(110,0)^{a}$ & $109,93 \pm 10,58(110,0)$ & $71,41 \pm 9,98(70,0)^{a}$ & $74,19 \pm 8,79(70,0)$ \\
\hline & {$[106,06-115,62]$} & {$[106,05-113,82]$} & {$[67,75-75,08]$} & {$[70,96-77,42]$} \\
\hline \multirow{2}{*}{$50 \mathrm{~min}$ rec } & $112,19 \pm 13,21(110,0)^{a}$ & $112,70 \pm 10,46(118,0)$ & $74,83 \pm 10,64(70,0)$ & $74,83 \pm 7,94(70,0)$ \\
\hline & {$[107,35-117,04]$} & {$[108,87-116,55]$} & {$[70,93-78,74]$} & {$[71,92-77,75]$} \\
\hline \multirow{2}{*}{$60 \mathrm{~min}$ rec } & $111,74 \pm 11,97(110,0)^{a}$ & $115,35 \pm 10,43(120,0)$ & $74,64 \pm 9,97(70,0)$ & $77,80 \pm 8,17(80,0)$ \\
\hline & {$[107,35-116,13]$} & {$[111,53-119,18]$} & {$[70,98-78,30]$} & {$[74,81-80,80]$} \\
\hline
\end{tabular}

Legenda: PAS = pressão arterial sistólica; PAD = pressão arterial diastólica; $\mathrm{mmHg}=$ milímetros de mercúrio; $\min =$ minuto; rec = recuperação. 
${ }^{a}$ Valor com diferença estatística em relação ao repouso (ANOVA para medidas repetidas seguida do teste de Bonferroni; $p<0,05$.

Tabela 5- Valores médios, seguidos dos seus respectivos desvios-padrão, mediana e intervalo de confiança a $95 \%$ de $\mathrm{SpO}_{2}$ e f obtidos nos protocolos controle e experimental em repouso e durante o período de recuperação.

\begin{tabular}{|c|c|c|c|c|}
\hline \multirow{2}{*}{ Tempo } & \multicolumn{2}{|c|}{$\mathrm{SpO}_{2}(\%)$} & \multicolumn{2}{|c|}{ f (irpm) } \\
\hline & Controle & Experimental & Controle & Experimental \\
\hline \multirow{2}{*}{ Repouso } & $97,12 \pm 0,88(97,0)$ & $97,45 \pm 0,67(97,0)$ & $16,25 \pm 3,41(16,0)$ & $15,74 \pm 2,90(16,0)$ \\
\hline & {$[96,80-97,45]$} & {$[97,20-97,69]$} & {$[15,00-17,51]$} & {$[14,67-16,80]$} \\
\hline \multirow{2}{*}{$1 \mathrm{~min} \mathrm{rec}$} & $96,64 \pm 1,05(97,0)^{\mathbf{b}}$ & $97,25 \pm 0,85(97,0)$ & $22,70 \pm 4,54(24,0)^{a}$ & $23,35 \pm 4,74(24,0)^{a}$ \\
\hline & {$[96,26-97,03]$} & {$[96,94-97,57]$} & {$[21,04-24,37]$} & [21,61 - 25,09] \\
\hline \multirow{2}{*}{$3 \mathrm{~min} \mathrm{rec}$} & $96,67 \pm 0,79(97,0)^{\mathbf{b}}$ & $97,32 \pm 0,70(97,0)$ & $20,90 \pm 3,53(20,0)^{a}$ & $21,67 \pm 3,83(24,0)^{a}$ \\
\hline & {$[96,38-96,96]$} & {$[97,06-97,58]$} & {$[19,60-22,19]$} & {$[20,27-23,08]$} \\
\hline \multirow{2}{*}{$5 \mathrm{~min}$ rec } & $96,74 \pm 0,81(97,0)^{\mathbf{b}}$ & $97,32 \pm 0,79(97,0)$ & $19,74 \pm 3,41(20,0)^{a}$ & $20,51 \pm 3,53(20,0)^{a}$ \\
\hline & {$[96,44-97,04]$} & {$[97,03-97,61]$} & {$[18,48-20,99]$} & {$[19,21-21,81]$} \\
\hline \multirow{2}{*}{$7 \mathrm{~min}$ rec } & $96,58 \pm 0,88(97,0)^{a, b}$ & $97,29 \pm 0,64(97,0)$ & $18,70 \pm 3,32(20,0)^{a}$ & $19,61 \pm 3,32(20,0)^{a}$ \\
\hline & {$[96,25-96,90]$} & {$[97,05-97,52]$} & {$[17,48-19,93]$} & {$[18,39-20,83]$} \\
\hline \multirow{2}{*}{$10 \mathrm{~min}$ rec } & $96,54 \pm 0,96(97,0)^{a, b}$ & $97,12 \pm 0,67(97,0)$ & $17,41 \pm 3,35(16,0)^{\mathbf{b}}$ & $19,22 \pm 3,33(20,0)^{a}$ \\
\hline & {$[96,19-96,90]$} & {$[96,88-97,37]$} & {$[16,18-18,65]$} & {$[18,00-20,44]$} \\
\hline \multirow{2}{*}{$20 \mathrm{~min}$ rec } & $96,38 \pm 0,98(96,0)^{\mathbf{a}, \mathbf{b}}$ & $97,12 \pm 0,95(97,0)$ & $17,16 \pm 3,29(16,0)$ & $18,06 \pm 3,24(20,0)^{a}$ \\
\hline & {$[96,02-96,75]$} & {$[96,77-97,48]$} & {$[15,95-18,37]$} & {$[16,87-19,25]$} \\
\hline \multirow{2}{*}{$30 \mathrm{~min}$ rec } & $96,45 \pm 0,96(96,0)^{a, b}$ & $97,51 \pm 0,85(98,0)$ & $16,90 \pm 3,04(16,0)$ & $17,16 \pm 2,95(16,0)$ \\
\hline & {$[96,09-96,80]$} & {$[97,20$ - 97,82] } & {$[15,78-18,02]$} & {$[16,07-18,24]$} \\
\hline \multirow{2}{*}{$40 \mathrm{~min}$ rec } & $96,80 \pm 0,87(97,0)^{\mathbf{b}}$ & $97,64 \pm 0,79(98,0)$ & $16,64 \pm 3,28(16,0)$ & $17,16 \pm 3,29(16,0)$ \\
\hline & {$[96,48-97,12]$} & {$[97,35$ - 97,93] } & {$[15,44-17,84]$} & {$[15,95-18,37]$} \\
\hline \multirow{2}{*}{$50 \mathrm{~min}$ rec } & $97,16 \pm 0,82(97,0)^{\mathbf{b}}$ & $97,61 \pm 0,61(98,0)$ & $15,61 \pm 2,98(16,0)$ & $16,77 \pm 2,99(16,0)$ \\
\hline & {$[96,86-97,46]$} & {$[97,38-97,83]$} & {$[14,51-16,70]$} & {$[15,67-17,87]$} \\
\hline \multirow{2}{*}{$60 \mathrm{~min}$ rec } & $97,09 \pm 0,87(97,0)^{\mathfrak{b}}$ & $97,80 \pm 0,87(98,0)$ & $15,09 \pm 2,67(16,0)$ & $15,74 \pm 2,90(16,0)$ \\
\hline & {$[96,77-97,41]$} & {$[97,48-98,12]$} & {$[14,11-16,07]$} & {$[14,67-16,80]$} \\
\hline
\end{tabular}

Legenda: $\mathrm{SpO}_{2}$ = saturação parcial de oxigênio; $\mathrm{f}$ = frequência respiratória; irpm = incursões respiratórias por minuto; $\min =$ minuto; rec = recuperação.

${ }^{a}$ Valor com diferença estatística em relação ao repouso (ANOVA para medidas repetidas seguida do teste de Bonferroni; $p<0,05)$; ${ }^{b}$ Valor com diferença estatística em relação ao mesmo momento do protocolo experimental (Teste $t$ de Student para dados não pareados; $p<0,05$ ).

\section{Discussão}

Os resultados deste estudo mostraram que a hidratação com solução hidroeletrolítica realizada durante e após uma atividade física submáxima de intensidade constante e longa duração, influenciou o comportamento dos parâmetros cardiorrespiratórios de jovens ao possibilitar, durante a sua realização, oscilações mínimas dessas variáveis e consequentemente, menor sobrecarga ao sistema e, durante a recuperação, retorno mais rápido das mesmas próximo às condições basais.

A avaliação da massa corporal dos sujeitos antes e ao final dos protocolos aplicados mostrou que a quantidade de líquido perdida no PC foi em média $2 \%$ do peso corporal, perda essa que foi evitada com a hidratação. Esses achados podem explicar a queda da PAS e da PAD observada durante o exercício quando nenhum fluido foi ingerido, e a manutenção de seus valores, quando a solução hidroeletrolítica foi administrada. Presume-se que a redução do volume sistólico ocasionada pela queda da volemia e consequente comprometimento do retorno venoso, condições que são observadas com o aumento do déficit hídrico (MERRY et al., 2010; RODRIGUEZ et al., 2009), seja responsável pelo comportamento da pressão arterial que foi encontrado no presente estudo.

Padrão semelhante ao observado para a PAS e para a PAD foi encontrado no estudo de González-Alonso et al. (1998). Apesar de permanecerem constantes durante os primeiros 90 minutos de exercício, as pressões arteriais reduziram significantemente a partir de duas 
horas de exercício desidratado, sendo esse comportamento não observado quando mantida a hidratação. Outro estudo observou redução significante a partir de uma hora de exercício da PAS e da pressão arterial média na condição desidratada comparada à condição hidratada Contudo, a PAD foi notavelmente constante em todo o exercício, sem qualquer influência da hidratação (GONZÁLEZ-ALONSO et al., 1995).

Contrapondo-se à queda da pressão arterial, os valores de FC, mesmo que não significantes, foram maiores quando nenhum fluido foi ingerido durante o exercício. Corroborando com esse achado, Hamilton et al. (1991) e González-Alonso et al. (1995) evidenciaram aumento da FC (10\% e $19 \%$ ), bem como redução do volume de ejeção $(15 \%$ e $28 \%)$ quando os sujeitos executavam duas horas de exercício sem o consumo de qualquer fluido. Quando água ou fluido a base de pó de gatorade foram administrados, a FC aumentou respectivamente, $5 \%$ e $6 \%$, e o volume de ejeção não se alterou (GONZÁLEZ-ALONSO et al., 1995; HAMILTON et al., 1991).

Khanna e Manna (2005), ao submeterem atletas a um esforço submáximo, observaram valores de FC menores quando bebida a base de carboidratos e eletrólitos foi ingerida a cada 15 minutos durante o exercício, ao contrário da não ingestão de solução. Contrariando esses achados, Horswill et al. (2008) não encontraram evidências de que a ingestão aguda de carboidratos antes e durante uma hora de exercício a $65 \%$ do $\mathrm{VO}_{2}$ máximo altera a resposta da FC como também da temperatura central.

Possivelmente, condições como alteração do metabolismo muscular, redução da sensibilidade dos barorreceptores (CHARKOUDIAN et al., 2003), dificuldade na manutenção da pressão arterial (GONZÁLES-ALONSO et al., 1997), aumento dos níveis de catecolaminas circulantes e maior hipertermia (GONZÁLEZ-ALONSO et al., 1995), possam ter influenciado o controle autonômico da FC (CARTER III et al., 2005) e estarem envolvidos com a sua elevação na condição hipoidratada.

Em relação ao comportamento observado para a $\mathrm{SpO}_{2}$, levando em consideração que todos os voluntários eram saudáveis e sem qualquer tipo de alteração respiratória, a queda observada na $\mathrm{SpO}_{2}$ quando comparado o repouso com o tempo de 90 minutos em ambos os protocolos sugere uma eventualidade sem implicações fisiológicas.
Entretanto, a incapacidade do sistema circulatório sustentar um aumento linear na distribuição de oxigênio para os músculos locomotores (MORTENSEN et al., 2005) pode estar implicada na queda da $\mathrm{SpO}_{2}$ ao final do exercício.

A recuperação da FC imediatamente após o exercício é mediada pela reativação na modulação da atividade parassimpática e a taxa de declínio desse parâmetro parece ser reflexo da recuperação mais rápida da atividade simpática necessária durante o exercício físico (IMAI et al., 1994). Adicionalmente, a taxa de retorno da FC aos valores basais após o exercício está associada à boa condição física e de saúde (SHETLER et al., 2001).

O seguimento da ingestão de solução hidroeletrolítica no período de pós-exercício promoveu, no presente estudo, melhor recuperação da FC em comparação à condição na qual nenhum fluido foi ofertado.

Tem sido sugerido que a hidratação promove redução da atividade simpática que, provavelmente, decorre de um efeito secundário da atividade vagal aferente reforçada em resposta à modulação dos barorreceptores durante a distensão gástrica (CHARKOUDIAN et al., 2003; YUN et al., 2005). Vianna et al. (2008) mostraram que o consumo de água acelerou a reativação cardíaca vagal pós-exercício, mas as respostas de FC não sofreram significante influência. Já Routledge et al. (2002) constataram que ingestão de $500 \mathrm{ml}$ de água provocou uma resposta bradicárdica seguida por um aumento da atividade cardíaca vagal. Esses aspectos podem ter influenciado o padrão de resposta da FC observado no presente estudo quando a solução hidroeletrolítica foi ingerida.

Adicionalmente, o aumento da temperatura corporal, desencadeado pelo exercício e pela hipoidratação, é conhecido por aumentar o disparo do nó sinusal (CRANDALL et al., 2000). Ainda, a hiperosmolaridade ocasionada pela desidratação, foi referida como provável causa do aumento da atividade simpática em pessoas desidratadas (CHARKOUDIAN e RABBITTS, 2009). Dessa maneira, a hidratação e a temperatura corporal do indivíduo podem alterar o grau do controle autonômico da FC durante a recuperação do exercício (CARTER III et al., 2005). 
No presente estudo, imediatamente após o exercício, o valor de temperatura corporal dos sujeitos na condição hipoidratada foi maior do que na condição hidratada. Partindo desse pressuposto, pode-se inferir que a pequena variação na temperatura corporal observada nos indivíduos hidratados associada à manutenção do estado de hidratação no pós-exercício também influenciaram o comportamento da FC observado.

Apesar da solução hidroeletrolítica ter proporcionado melhor recuperação da FC, não foi observado retorno de seus valores às condições basais. Khanna e Manna (2005) após administrarem $100 \mathrm{ml}$ de bebida contendo carboidratos e eletrólitos ao longo de 20 minutos pós-exercício submáximo evidenciaram que, apesar da melhora significante da FC, não observou retorno desse parâmetro aos valores pré-exercício. Presume-se que o tempo de recuperação utilizado no estudo de Khanna e Manna (2005) (20 minutos), bem como no presente estudo (60 minutos), não foi suficiente para total restauração da função cardiovascular, observada apenas em 120 minutos por $\underline{\text { Costill e }}$ Sparks (1973).

Em relação ao comportamento da PAS, os maiores valores observados nos minutos iniciais da recuperação em relação ao repouso, em ambos os protocolos, parece estar associado ao término imediato do exercício físico, uma vez que o mesmo exige do organismo capacidade em coordenar múltiplas respostas metabólicas frente ao aumento das necessidades da musculatura esquelética em atividade (LE et al., 2008).

Já os valores de PAD mantiveram-se constantes quando houve a reposição líquida e exibiram queda significante nos primeiros minutos da recuperação no $P C$. A redução da $P A D$ na condição hipoidratada pode estar relacionada ao aumento da temperatura corporal evidenciado após o exercício físico. A fim de auxiliar o processo de dissipação do calor, o sistema cardiovascular reduz a resistência periférica promovendo vasodilatação com concomitante aumento do fluxo sanguíneo cutâneo (KURZ, 2008).

Ainda assim, a hidratação teve pouco efeito sobre os valores de pressão arterial, podendo ser consideradas ocasionais as diferenças encontradas ao longo do período de recuperação. Brown et al. (2005), ao avaliarem as respostas cardiovasculares à água potável, observaram que ao longo de uma hora, a ingestão do líquido promoveu pouco efeito sobre a pressão arterial em jovens saudáveis.
Contrapondo-se às repostas de pressão arterial observadas neste estudo, a $\mathrm{SpO}_{2}$ foi significantemente influenciada pela hidratação no período pós-exercício. González-Alonso e Calbet (2003) evidenciaram que o estresse no calor reduz $\circ \mathrm{VO}_{2}$ máximo, acelera a queda do débito cardíaco e da pressão arterial média, que levam ao decréscimo do fluxo sanguíneo muscular e da oferta de oxigênio. Provavelmente, a preservação do sistema circulatório dos indivíduos hidratados tenha beneficiado o comportamento da $\mathrm{SpO}_{2}$ observado neste estudo.

Por fim, o comportamento da $f$ não foi influenciado pela condição de hidratação e mostrou-se dentro dos padrões de normalidade. Os maiores valores encontrados nos primeiros minutos da recuperação comparados ao repouso são esperados, visto que estímulos físicos e químicos que ocorrem com o exercício, como a diminuição do $\mathrm{pH}$ e o aumento da temperatura, promovem a elevação da frequência respiratória (HAYASHI et al., 2006).

\section{Conclusões}

O protocolo de hidratação empregado promoveu, durante o exercício, manutenção dos valores de PAS e PAD e menor incremento da FC, não sendo observadas alterações significantes na $\mathrm{SpO}_{2}$; e, ao longo do período de recuperação, promoveu retorno mais rápido da FC próximo às condições basais, bem como conservou, nesse mesmo período, os valores de PAS (a partir do quinto minuto), $\mathrm{PAD}, \mathrm{SpO}_{2}$ e f (a partir do trigésimo minuto).

O protocolo de hidratação aplicado no estudo, ao amenizar as alterações cardiorrespiratórias induzidas pelo exercício e acelerar a sua recuperação, contribui para encorajar os indivíduos que praticam atividades físicas a seguir uma rotina profilática de hidratação que pode reduzir o risco de distúrbios associados às alterações hemodinâmicas e/ou desequilíbrios eletrolíticos.

\section{Referências}

ARMSTRONG, L. E. Hydration assessment techniques. Nutrition Reviews, Malden, v. 63, p. S40-S54, 2005.

BROWN, C. M.; BARBERINI, L.; DULLOO, A. G.; MONTANI, J.-P. Cardiovascular responses to water drinking: does osmolality play a role?

American Journal of Physiology Regulatory Integrative and Comparative Physiology, Fribourg, v. 289, n. 6, p. R1687-R1692, 2005. 
CARTER III, R.; CHEUVRONT, S. N.; WRAY, D. W.; KOLKA, M. A.; STEPHENSON, L. A.; SAWKA, M. N. The influence of hydration status on heart rate variability after exercise heart stress. Journal of Thermal Biology, New York, v. 30, n. 7, p. 495-502, 2005.

CHARKOUDIAN, N.; HALLIWILL, J. R.; MORGAN, B. J.; EISENACH, J. H.; JOYNER, M. $\mathrm{J}$. Influences of hydration on postexercise cardiovascular control in humans. The Journal of Physiology, Bethesda, v. 552, p. 635-644, 2003.

CHARKOUDIAN, N.; RABBITTS, J. A. Sympathetic neural mechanisms in human cardiovascular health and disease. Mayo Clinic Proceedings, Oxford, v. 84, n. 9, p. 822-830, 2009.

COFFEY, V.; LEVERITT, M.; GILL, N. Effect of recovery modality on 4-hour repeated treadmill running performance and changes in physiological variables. Journal of Science and Medicine in Sport, Bethesda, v. 7, n. 1, p. 1-10, 2004.

COSTILL, D. L.; SPARKS, K. E. Rapid fluid replacement following thermal dehydration. Journal of Applied Physiology, Muncie, v. 34, n. 3, p. 299-303, 1973.

CRANDALL, C. G.; ZHANG, R.; LEVINE, B. D. Effects of whole body heating on dynamic baroreflex regulation of heart rate in humans. American Journal of Physiology Heart and Circulatory Physiology, Dallas, v. 279, n. 5, p. H2486-H2492, 2000.

GISOLFI, C. V.; DUCHMAN, S. M. Guidelines for optimal replacement beverages for different athletic events. Medicine and Science in Sports \& Exercise, lowa City, v. 24, p. 679-687, 1992.

GLAISTER, M. Multiple sprint work physiological responses, mechanisms of fatigue and the influence of aerobic fitness. Sports

Medicine, Twickenham, v. 35, n. 9, p. 757-777, 2005.

GONZÁLEZ-ALONSO, J.; CALBET, J. A. Reductions in systemic and skeletal muscle blood flow and oxygen delivery limit maximal aerobic capacity in humans. Circulation, Hagerstown, v. 107, n. 6, p. 824-830, 2003.

GONZÁLEZ-ALONSO, J.; CALBET, J. A.; NIELSEN, B. Muscle blood flow is reduced with dehydration during prolonged exercise in humans. The Journal of Physiology, Bethesda, v. 513, p. 895-905, 1998.

GONZÁLEZ-ALONSO, J.; MORA-RODRIGUEZ, R.; BELOW, P. R.; COYLE, E. F. Dehydration markedly impairs cardiovascular function in hyperthermic endurance athletes during exercise. Journal of Applied Physiology, Bethesda, v. 82, n. 4, p. 1229-1236, 1997.

GONZÁLEZ-ALONSO, J.; MORA-RODRIGUEZ, R.; BELOW, P. R.; COYLE, E. F. Dehydration reduces cardiac output and increases systemic and cutaneous vascular resistance during exercise. Journal of Applied Physiology, Bethesda, v. 79, n. 5, p. 1487-1496, 1995.

HAMILTON, M. T.; GONZALEZ-ALONSO, J.; MONTAIN, S. J.; COYLE, E. F. Fluid replacement and glucose infusion during exercise prevent cardiovascular drift. Journal of Applied Physiology, Bethesda, v. 71, n. 3, p. 871-877, 1991.

HAYASHI, K.; HONDA, Y.; OGAWA, T.; KONDO, N.; NISHIYASU, T. Relationship between ventilatory response and body temperature during prolonged submaximal exercise. Journal of Applied Physiology, Bethesda, v. 100, n. 2, p. 414-420, 2006.

HERNANDEZ, A. J.; NAHAS, R. M. Modificações dietéticas, reposição hídrica, suplementos alimentares e drogas: comprovação de ação ergogênica e potenciais riscos para a saúde. Revista Brasileira de Medicina do Esporte, Niterói, v. 15, n. 3, p. 3-12, 2009.

HORSWILL, C. A. ; STOFAN, J. R. ; LOVETT, S. C. ; HANNASCH, C. Core temperature and metabolic responses after carbohydrate intake during exercise at 30 degrees $C$. Journal of Athletic Training, Barrington, v. 43, n. 6, p. 585591, 2008.

IMAI, K.; SATO, H.; HORI, M.; KUSUOKA, H.; OZAKI, H.; YOKOYAMA, H.; TAKEDA, H.; INOUE, M.; KAMADA, T. Vaguely mediated heart rate recovery after exercise is accelerated in athletes but blunted in-patients with chronic heart failure. Journal of American College of Cardiology, New York, v. 24, p. 1529-1535, 1994.

KHANNA, G.L.; MANNA, I. Supplementary effect of carbohydrate-electrolyte drink on sports performance, lactate removal \& cardiovascular response of athletes. Indian Journal of Medical Research, New Delhi, v. 121, n. 5, p. 665-669, 2005.

KURZ, A. Physiology of thermoregulation. Best Practice \& Research Clinical Anaesthesiology, Amsterdam, v. 22, n. 4, p. 627-644, 2008.

LE, V. V.; MITIKU, T.; SUNGAR, G.; MYERS, J.; FROELICHER, V. The blood pressure response to dynamic exercise testing: a systematic review. Progress in Cardiovascular Diseases, Philadelphia, v. 51, n. 2, p. 135-160, 2008. 
MARTINMÄKI, K.; RUSKO, H. Time-frequency analysis of heart rate variability during immediate recovery from low and high intensity exercise.

European Journal of Applied Physiology, Berlin, v. 102, n. 3, p. 353-360, 2008.

MERRY, T. L.; AINSLIE, P. N.; COTTER, J. D. Effects of aerobic fitness on hypohydrationinduced physiological strain and exercise impairment. Acta Physiologica, Dunedin, v. 198, n. 2, p. 179-190, 2010.

MONTAIN, S. J. Hydration recommendations for sport 2008. Current Sports Medicine Reports, Natick, v. 7, n. 4, p. 187-192, 2008.

MONTAIN, S. J.; COYLE, E. F. Fluid ingestion during exercise increases skin blood flow independent of increases in blood volume.

Journal of Applied Physiology, Bethesda, v. 73, p. 903-910, 1992.

MORTENSEN, S. P.; DAWSON, E. A.; YOSHIGA, C. C.; DALSGAARD, M. K.; DAMSGAARD, R.; SECHER, N. H.; GONZALEZ-ALONSO, J. Limitations to systemic and locomotor limb muscle oxygen delivery and uptake during maximal exercise in humans. The Journal of Physiology, Bethesda, v. 566, p. 273-258, 2005.

PARDINI, R.; MATSUDO, S.; ARAUJO, T.; MATSUDO, V.; ANDRADE, E.; BRAGGION, G.; ANDRADE, D.; OLIVEIRA, L.; FIGUEIRA, A.; RASO, V. Validação do questionário internacional de nível de atividade física (IPAQ - versão 6): estudo piloto em adultos jovens brasileiros.

Revista Brasileira de Ciência e Movimento, Brasília, v. 9, n. 3, p. 45-51, 2001.

RODRIGUEZ, N. R.; DIMARCO, N. M.; LANGLEY, S. American College of Sports Medicine position stand: nutrition and athletic performance. Medicine and Science in Sports \& Exercise, Hagerstown, p. 709-731, 2009.

ROUTLEDGE, H. C.; CHOWDHARY, S.; COOTE, J. H.; TOWNEND, J. N. Cardiac vagal response to water ingestion in normal human subjects.

Clinical Science, Birmingham, v. 103, n. 2, p. 157-162, 2002.

SHETLER, K.; MARCUS, R.; FROELICHER, V. F.; VORA, S.; KALISETTI, D.; PRAKASH, M.; DO, D.; MYERS, J. Heart rate recovery: validation and methodologic issues. Journal of American

College of Cardiology, New York, v. 38, p. 19801987, 2001.

TEBEXRENI, A. S.; LIMA, E. V.; TAMBEIRO, V. L.; BARROS NETO, T. L. de Protocolos tradicionais em ergometria, suas aplicações práticas "versus" protocolo de rampa. Revista da
Sociedade de Cardiologia do Estado de São

Paulo, São Paulo, v. 11, n. 3, p. 519-528, 2001.

VI DIRETRIZES BRASILEIRAS DE HIPERTENSÃO. Arquivos Brasileiros de Cardiologia, São Paulo, v. 95, n. 1, p. 1-51, 2010.

VIANNA, L. C.; OLIVEIRA, R. B.; SILVA, B. M.; RICARDO, D. R.; ARAUJO, C. G. S. Water intake accelerates post-exercise cardiac vagal reactivation in humans. European Journal of Applied Physiology, Berlin, Rio de Janeiro, v. 102, n. 3, p. 283-288, 2008.

VON DUVILLARD, S. P.; BRAUN, W. A.; MARKOFSKI, M.; BENEKE, R.; LEITHAUSER, R. Fluids and hydration in prolonged endurance performance. Nutrition, Burbank, v. 20, n. 7-8, p. 651-656, 2004.

YUN, A. J.; LEE, P. Y.; BAZAR, K. A. Clinical benefits of hydration and volume expansion in a wide range of illnesses may be attributable to reduction of sympatho-vagal ratio. Medical Hypotheses, New York, v. 64, n. 3, p. 646-650, 2005.

Apoio Financeiro: Fapesp.

\author{
Endereço: \\ Luiz Carlos Marques Vanderlei \\ FCT/UNESP, Departamento de Fisioterapia \\ Rua Roberto Simonsen, 305 \\ Cidade Universitária \\ Presidente Prudente SP Brasil \\ 19060-900 Caixa Postal 957 \\ Telefone: (18) 3229.5388 Ramal 5365 \\ Fax: (18) 3221.5897. \\ E-mail: Icmvanderlei@fct.unesp.br
}

Recebido em: 17 de dezembro de 2010. Aceito em: 21 de setembro de 2011.

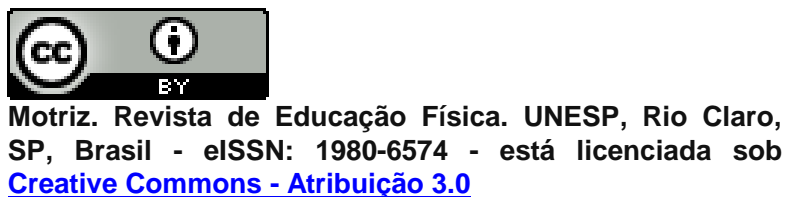
Creative Commons - Atribuição 3.0 